\title{
Questionnaire-Based Research on Opinions of Visitors for Communication Robots at an Exhibition in Japan
}

\author{
Tatsuya Nomura ${ }^{1,2}$, Takugo Tasaki ${ }^{2,3}$, Takayuki Kanda ${ }^{2}$, \\ Masahiro Shiomi ${ }^{2}$, Hiroshi Ishiguro ${ }^{2}$, and Norihiro Hagita ${ }^{2}$ \\ 1 Department of Media Informatics, Ryukoku University, \\ 1-5, Yokotani, Setaohe-cho, Otsu, Shiga 520-2194, Japan \\ 2 ATR Intelligent Robotics and Communication Laboratories, \\ 2-2, Hikaridai, Seika-cho, Soraku-gun, Kyoto 619-0288, Japan \\ 3 Graduate School of Corporate Information, Hannan University, \\ 5-4-33, Amamihigashi, Matsubara, Osaka 580-8502, Japan
}

\begin{abstract}
This paper reports the results of questionnaire-based research conducted at an exhibition of interactive humanoid robots that was held at the Osaka Science Museum, Japan. The aim of this exhibition was to investigate the feasibility of communication robots connected to a ubiquitous sensor network, under the assumption that these robots will be practically used in daily life in the not-so-distant future. More than ninety thousand people visited the exhibition. A questionnaire was given to the visitors to explore their opinions of the robots. Statistical analysis was done on the data of 2,301 respondents. It was found that the visitors' opinions varied according to age; younger visitors did not necessarily like the robots more than elderly visitors; positive evaluation of the robots did not necessarily conflict with negative evaluations such as anxiety; there was no gender difference; and there was almost no correlation between opinions and the length of time spent near the robots.
\end{abstract}

\section{Introduction}

The aim of communication robots is to act in environments with humans and assist humans through communication with them. Humanoid-type robots are considered to be useful in this communication task by, for example, gesturing with their faces, arms, and eyes in guidance tasks for maps.

One method of implementing communication robots is ubiquitous computing, where robots use information from sensors, not only in the robots themselves but also in the environments in which they exist [1 233. This method assumes that all of the objects in the environments have their own IDs by using wireless tag systems 4 |5|6. The most important characteristic of this method is its reduced computational cost for identification of environments by robots, which is difficult in cases where each robot must act alone.

Guidance in museums is considered an effective application of communication robots using these ubiquitous sensor networks. Although research on this task 
has been considered for one robot, it focused on providing information by the robot 7 . From the perspective of communication robots, the interaction between robots and humans via sensor network information is more important.

To investigate the effectiveness of communication robots connected through ubiquitous sensor networks in guidance tasks, an exhibition of humanoid robots, called "Robovie" [8, was held at the Osaka Science Museum 1, Japan, for approximately two months in 2004. At this exhibition, a questionnaire was distributed to visitors to explore their opinions of the robots.

Although there has been some research on psychological evaluations by visitors of robots at science museums 9]10]11, these studies have been limited to individual impressions of specific robots behaving alone. The Osaka Science Museum exhibition focused on interaction between visitors and robots via sensor network information in a guidance task. Thus, the visitors' opinions of the robots are assumed to reflect impressions of this interaction. In particular, the research evaluates not only interest in, friendliness toward, and perceives effectiveness of the robots but also anxiety toward them. In addition, it focuses on relations among these psychological features, concrete behavior such as time spent near the robots, and personal traits such as gender and age. Anxiety toward robots and this feeling's relation to behaviors and personal traits are important factors to investigate when communication robots behave in environments with humans and communicate with them [12].

This paper gives an overview of the communication robot exhibition and then analyzes the results of the questionnaires gathered there.

\section{The Communication Robots Exhibition}

This section presents an overview of the ubiquitous sensor network, the communication robots, the procedures, and the questionnaire used at the communication robots exhibition.

\subsection{Overview of Systems}

The ubiquitous sensor network was constructed on the 4th floor of the Osaka Science Museum (see Fig. 1). This sensor network records visitor behaviors, and the information obtained was used by the robots to assist visitors in viewing exhibits at the museum and to encourage their interest in science and technology.

Sensor Systems: In this exhibition, visitors had wireless tags. Signals from these tags were detected by using 20 wireless tag readers. A tag reader can detect signals from tags within a maximum of $10 \mathrm{~m}$. The interpolation of the strengths of signals detected by several tag readers makes it possible to determine the physical positions of tags. Eighteen wireless tag readers were hung on the ceiling near exhibits to detect whether visitors stayed near the exhibits. Two tag readers were installed into the robots.

${ }^{1}$ http://www.sci-museum.kita.osaka.jp/ 


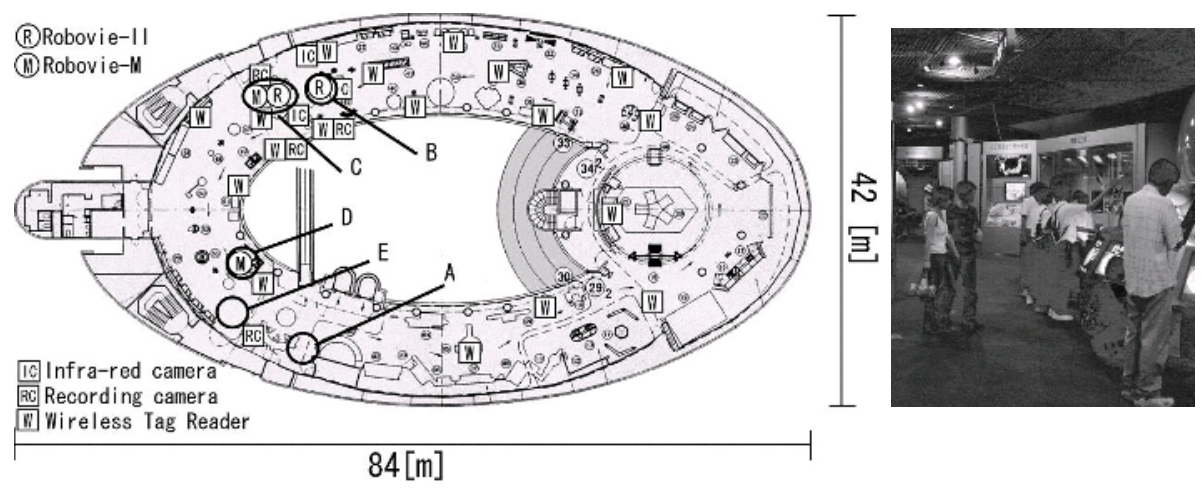

Fig. 1. Overview of the Osaka Science Museum and a Visitor Scene
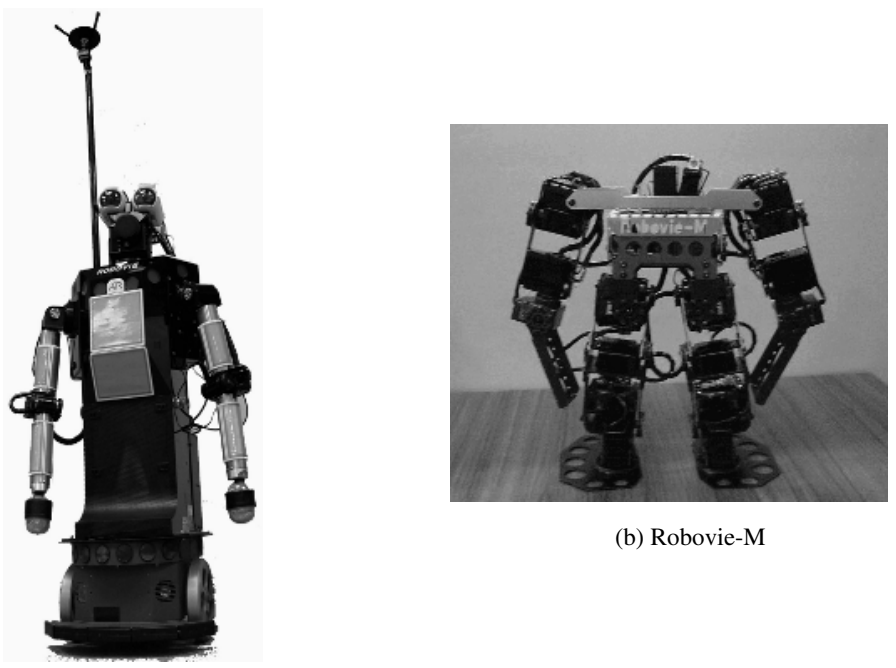

(b) Robovie-M

(a) Robovie-II

Fig. 2. Two Types of Robots Used in the Exhibition: (a) Human-Sized Robot "Robovie-II", (b) Small-Sized Robot "Robovie-M"

In addition, three infrared cameras were assigned to detect positions of the robots and four digital cameras were assigned to record scenes at the exhibition. Each of the cameras and tag readers, except for the ones assigned to the robots, was connected to a PC to control information maintained in a database processed on a central server via ethernet.

Robots: In the exhibition, two types of communication robots were used. Figure 2 shows the humanoid robot "Robovie" [8].

"Robovie-II", shown in Fig. 2(a), is a human-sized robot that stands $120 \mathrm{~cm}$ tall. Its diameter is $40 \mathrm{~cm}$, and it weighs about $40 \mathrm{~kg}$. The robot has two arms 
$(4 \times 2$ DOF (degrees of freedom) $)$, a head $(3 \mathrm{DOF})$, two eyes $(2 \times 2 \mathrm{DOF}$ for gaze control), and a mobile platform (two driving wheels and one free wheel). This robot has various sensors, including skin sensors covering the entire body, 10 tactile sensors located around the mobile platform, an omni-directional vision sensor, two microphones to listen to human voices, and 24 ultra-sonic sensors for detecting obstacles. It carries a Pentium III PC on board for processing sensory data and generating gestures, including utterances. It is assigned one wireless tag reader.

"Robovie-M", shown in Fig. 2(b), is a small- sized robot that stands $29 \mathrm{~cm}$ tall. It has 22 DOF, which allows it to execute various gestures such as walking, bowing, and a handstand (see http://www.vstone.co.jp/top/p_info/ robot/robovie-m.html). Since the robot does not have its own function to make utterances, its utterances are performed by the connected PC.

\subsection{Procedures}

Flow of Visitors: Visitors experienced the exhibition as follows.

First, visitors register for their wireless tags at the reception desk at the entrance to the 4th floor (position A in Fig. 1). At this stage, their names, ages, and birthdates are registered and input to the ID tags provided them. Then, the registered names are automatically transfered into speech information that the robots use in their utterances to visitors.

Visitors are then free to observe exhibits in the museum. All of the wireless tag information is recorded in the database. While viewing the exhibits, visitors interact with robots, each of which has its own role. One provides guidance of the exhibits while moving alone. Two of them communicate with each other to provide guidance of the exhibits (position $\mathrm{C}$ in Fig. 1). The other robot executes interaction behaviors, such as calling visitors' names near the exit (position D in Fig. 1).

When visitors finish viewing the exhibits, they are asked to freely respond to a questionnaire on their opinions of the robots and the exhibition at the exit (position E in Fig. 1). The wireless tags are then returned.

Roles of the Robots: At the exhibition, two Robovie-IIs and two Robovie-Ms were used.

One Robovie-II executed exhibit guidance in the museum while moving about (Fig. 3(a)). It explained the contents of exhibits, such as their history.

The other Robovie-II and one Robovie-M executed exhibit guidance while simulating interaction between them by synchronization via the network (Fig. 3(b)). Specifically, the Robovie-M explained an exhibit, the Robovie-II asked questions about it, and finally the Robovie-M responded to the question. Furthermore, these robots interacted with visitors by using information from the ubiquitous sensor network.

The remaining Robovie-M did not provide guidance but instead interacted with visitors by calling their names based on visitor tags and registered information, saying good-bye, asking visitors to return their tags, and so on (Fig. 3(c)). 


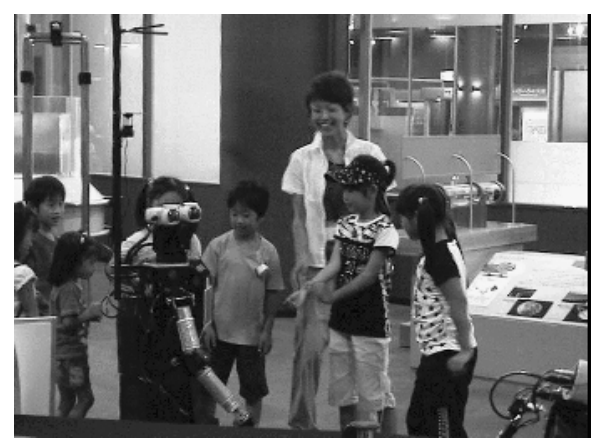

(a)

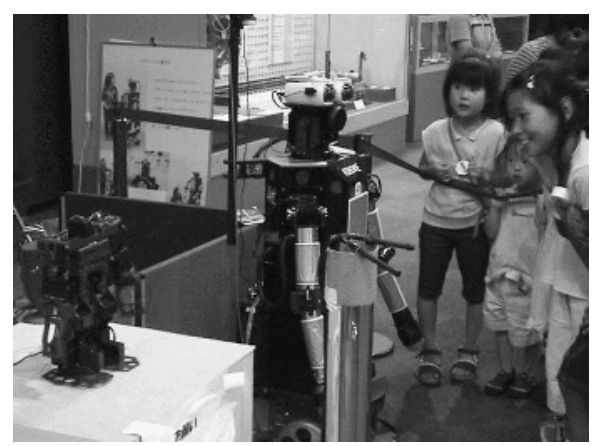

(b)

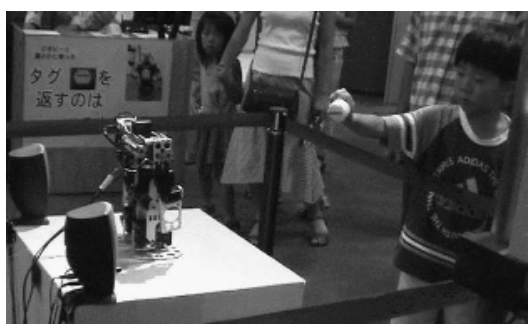

(c)

Fig. 3. Scenes of Interaction between the Robots and Visitors: (a) Guidance by Robovie-II, (b) Interaction between Robovie-II and Robovie-M, (c) Interaction with Robovie-M near the Exit

Questionnaire Items: The questionnaire used in the exhibition consisted of the following statements. Respondents indicated the degree to which each statement applies to them by marking whether they (1) "strongly agree", (2) "agree", (3) "are undecided", (4) "disagree", or (5) "strongly disagree".

\section{Item 1 (Interest):}

I am interested in the robots.

\section{Item 2 (Friendliness):}

I felt friendly toward the robots when I faced them.

\section{Item 3 (Effectiveness):}

I find guidance provided by the robots effective.

\section{Item 4 (Anxiety toward Interaction):}

I felt anxiety when the robots talked to me.

\section{Item 5 (Anxiety toward Social Influence):}

I feel anxiety about the possible widespread application of robots to perform tasks such as those shown at the exhibition in the near future.

The 1st, 2nd, and 3rd items measure respondents' interest in the robots, friendliness toward the robots, and evaluation of the robots' effectiveness, respectively. The 4th and 5th items measure the respondents' anxiety toward interaction with the robots and the social influence of the robots, respectively. 
The questionnaire also includes items on gender and age. The item on age has seven graded answers (for respondent age categories from the 10's to 70's). In addition, the questionnaire has an item for freely describing opinions about the robots and the exhibition.

\section{Analysis of Data}

The communication robots exhibition was held at the Osaka Science Museum, Japan, from July to August, 2004. This period included the Japanese summer holidays. By the end of the two-month period, the number of visitors reached 91,107 and the number of visitors who wore wireless tags was 11,927.

The total number of returned questionnaires was 3,034, the number of those including all of the five items shown in section 2.2 was 2,891 , and the number of those including both the gender item and the age item was 2,301. Analysis considering factors of age and gender was executed for these 2,301 samples. Finally, the number of questionnaires that included freely described opinions about the robots and the exhibition was 293 .

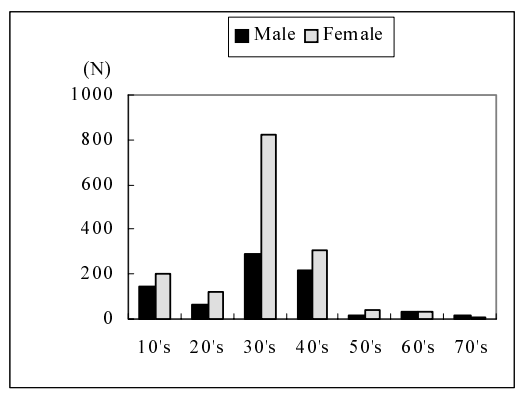

(a)

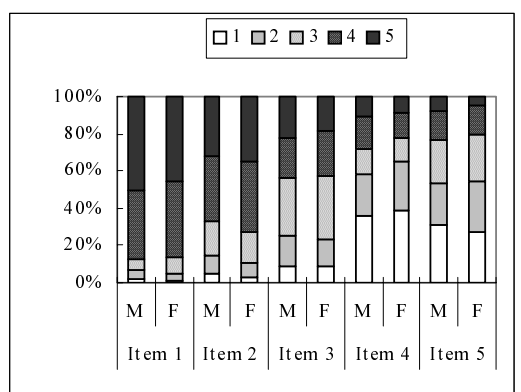

(b)

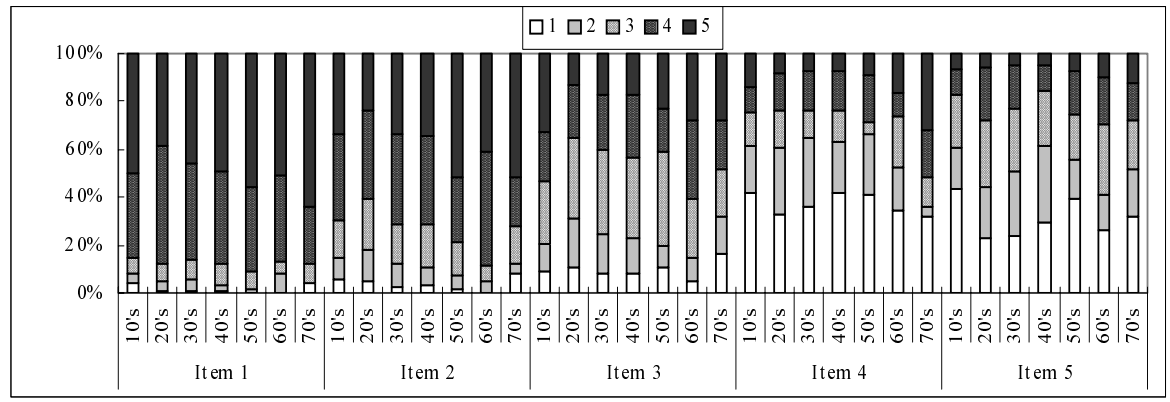

(c)

Fig. 4. Distributions of Respondents and Item Scores: (a) Distribution of Respondents based on Gender and Age, (b) Distribution of Item Scores based on Gender, (c) Distribution of Item Scores based on Age 
Answers were scored in reverse order of their listing, from 1 ("strongly disagree") to 5 ("strongly agree).

Moreover, the following information was measured as a behavior index, based on tag information from the ubiquitous sensor network:

T3: Time that visitors stayed within $3 \mathrm{~m}$ of the point where Robovie-II and Robovie-M simulated their communication.

The relationship between this behavior index and the item scores was also analyzed.

\subsection{Item Scores}

The number of male respondents was 777 and that of female respondents was 1,524. Moreover, the number of respondents aged in the 10's was 349, that in the 20 's was 182 , that in the 30 's was 1109 , that in the 40 's was 519 , that in the 50's was 56 , that in the 60 's was 61 , and that in the 70 's was 25 . Figure 4 (a) shows the distribution of respondents based on gender and age. This figure

Table 1. Mean Scores and Standard Deviations of Items based on Gender and Age, and Results of Two-Way ANOVA for the Item Scores

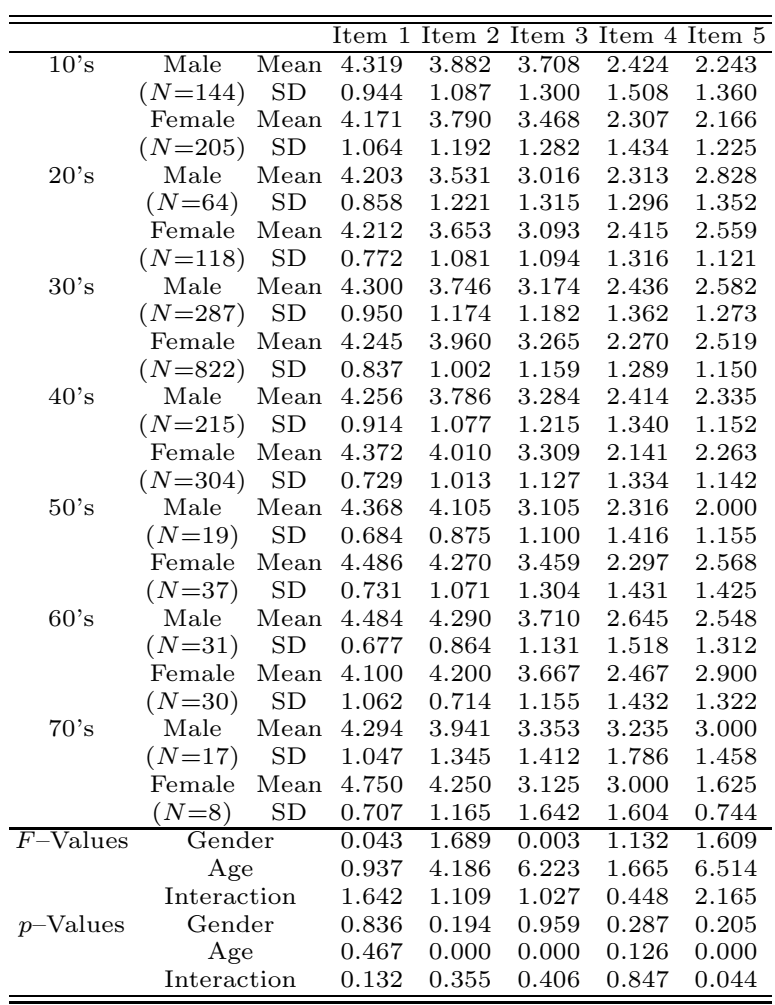


indicates that there was a bias among respondents aged in their 30's and 40's, in particular, females in their 30's.

Figures 4(b) and (c) show the distributions of the item scores based on gender and age, respectively. These figures show that the rates of respondents scoring more than 4 on items 1 and 2 were more than $80 \%$ and about $70 \%$, respectively. Moreover, the rates of the respondents scoring less than 2 on items 4 and 5 were each about $60 \%$. On the other hand, these figures imply that the distributions of the item scores may differ between ages.

Table 1 shows mean scores and standard deviations of the items based on gender and age, and the results of a two-way ANOVA for the item scores with factors of gender and age. There were statistically significant differences for items 2,3 , and 5 between ages. There was no statistically significant difference between genders. A Tukey post-hoc test obtained the following results:

- The scores of item 2 in the 20's group were lower than those in the 30's, 40's, 50 's and 60's groups.

- The scores of item 3 in the 20's group were lower than those in the 10's and 60 's groups. Moreover, those in the 10's group were higher than those in the 30's and 40's groups.

- The scores of item 5 in the 10's group were lower than those in the 20's, 60's, and 70's groups. Moreover, those in the 40's group were lower than those in the 20's and 30's groups.

\subsection{Time That Respondents Stayed Near the Robots}

The behavior index T3 may reflect the respondents' interest, friendliness, and anxiety toward the robots to some extent. However, it can also be influenced by external factors, such as congestion on the floor. In fact, the number of visitors per day was widely distributed during the period (maximum: 3,240, minimum: 767 , average: 1,898 , median: 1,780 ), due to the fact that this period included the Japanese summer holidays. Thus, the days that more than 2,250 people visited, including summer holidays, were assumed to be congested days and the effect of congestion on the behavior index was analyzed.

First, a two-way ANOVA with factors of the congestion condition and age was executed. Only the congestion condition had an effect (age: $F=1.186$, $p=0.083$; congestion: $F=20.406, p=0.000$; interaction: $F=0.885, p=0.505)$.

Next, a two-way ANOVA with factors of congestion and gender was executed. Both congestion and gender had an effect (gender: $F=8.111, p=0.004$; congestion: $F=44.930, p=0.000$; interaction: $F=1.171, p=0.279$ ). Figure 5(a) shows the mean values and standard deviations of T3 on the gender and congestion conditions. It was found that the T3 values of the visitors on congested days were about $50 \mathrm{sec}$ larger than those on non-congested days, and the T3 values of the female respondents were more than $10 \mathrm{sec}$ larger than those of the male visitors. 


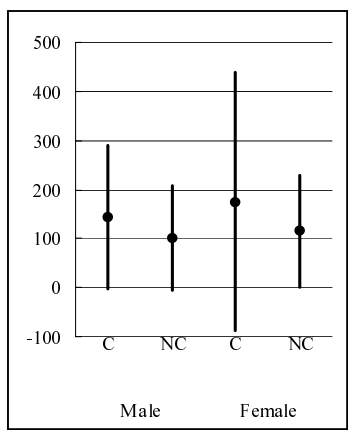

(a)

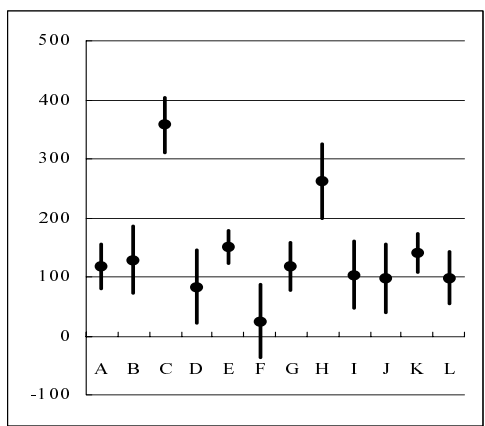

(b)

Fig. 5. Mean Values and Standard Deviations of T3: (a) on Gender and Congestion Condition (C: Congestion, NC: Non-Congestion, Male-C: $N=414$, Male-NC: $N=$ 363, Female-C: $N=699$, Female-NC: $N=825$ ), (b) on Categories of Freely Described Opinions (A: $N=14, \mathrm{~B}: N=6, \mathrm{C}: N=9, \mathrm{D}: N=5, \mathrm{E}: N=26, \mathrm{~F}: N=5, \mathrm{G}: N=12$, $\mathrm{H}: N=5, \mathrm{I}: N=6, \mathrm{~J}: N=6, \mathrm{~K}: N=18, \mathrm{~L}: N=10)$

Table 2. Peason's Correlation Coefficient $r$ between the Item Scores and Behavior Index T3

\begin{tabular}{|c|c|c|c|c|c|}
\hline & \multicolumn{5}{|c|}{ Item 1 Item 2 Item 3 Item 4 Item 5 T3 } \\
\hline$\overline{\text { Item } 1}$ & 0.521 & 0.385 & -0.095 & -0.123 & 0.077 \\
\hline & 0.521 & 9 & -0 & 28 & 0.059 \\
\hline Item 3 & 0.385 & - & -0.030 & -0.100 & 0.036 \\
\hline Ite & $-0.095-0.142$ & -0.030 & - & 0.372 & 0.048 \\
\hline te & $-0.123-0.128$ & -0.100 & 0.372 & - & -0.018 \\
\hline
\end{tabular}

\subsection{Correlations Between Item Scores and Behavior Index}

Table 2 shows Peason's correlation coefficient $r$ between the item scores and behavior index T3. There were medium-level correlations between items 1-3 and between items $4-5$. On the other hand, there were few correlations or low-level correlations between the group of items 1-3 and that of items 4-5. Moreover, there were few correlations between the item scores and behavior index T3.

\subsection{Freely Described Opinions}

A total of 293 sentences expressing opinions on the robots and exhibition were manually classified into several categories based on the contents of the sentences. This classification was executed by two people who discussed the contents of the sentences and categories until they reached a consensus in their classification results. Finally, 16 categories were established and each sentence was classified into one of them. Table 3 shows these categories, the number of sentences classified into each category, and examples of the sentences classified into each category. 
Table 3. Categories of Freely Described Opinions of the Robots and Exhibition, the Number of Opinions Classified into Each Category, and Examples of Opinions Classified into Each Category

\begin{tabular}{|c|c|}
\hline Category & $\bar{N}$ \\
\hline $\begin{array}{l}\text { A. Positive Opinions of the Robots Themselves } \\
\text { (Example: "I was glad to have the robots talk me.") }\end{array}$ & $\overline{23}$ \\
\hline $\begin{array}{l}\text { B. Expectations for Robots and Technology in the Future } \\
\text { (Example: "I would enjoy it if there were more kinds of robots."), }\end{array}$ & 16 \\
\hline $\begin{array}{l}\text { C. Positive Attitudes of Children toward the Robots } \\
\text { (Example: "My child seemed to be glad to be called by the robots.") }\end{array}$ & 17 \\
\hline $\begin{array}{l}\text { D. Desires on Interaction or Touch with the Robots } \\
\text { (Example: "I wanted to talk with the robots more.") }\end{array}$ & 8 \\
\hline $\begin{array}{l}\text { E. Negative Opinions of Communication with the Robots } \\
\text { (Example: "The robots' utterances were hard to listen to.") }\end{array}$ & 59 \\
\hline $\begin{array}{l}\text { F. Negative Emotions toward the Robots } \\
\text { (Example: "I felt a little fear toward the robots.") }\end{array}$ & 10 \\
\hline $\begin{array}{l}\text { G. Fear of Children toward the Robots } \\
\text { (Example: "My child seemed to feel fear toward the robots.") }\end{array}$ & 20 \\
\hline $\begin{array}{l}\text { H. Children's Indifference to or Non-Interest in the Robots } \\
\text { (Example: "My child seemed to lose interest in the robots } \\
\text { because they did not react to the name tag.") }\end{array}$ & 9 \\
\hline $\begin{array}{l}\text { I. Other Dissatisfaction with the Robots } \\
\text { (Example: "The robots' reactions were slower than what I expected.") }\end{array}$ & 12 \\
\hline $\begin{array}{l}\text { J. Physical Danger in Interaction with the Robots } \\
\text { (Example: "The robot's arm struck my child.") }\end{array}$ & 7 \\
\hline $\begin{array}{l}\text { K. Positive Evaluation of the Exhibition } \\
\text { (Example: "I was happy } \\
\text { because I could directly come in contact with the robots.") }\end{array}$ & 27 \\
\hline $\begin{array}{l}\text { L. Critical Requests for the Contents of the Exhibition } \\
\text { (Example: "Please prepare more kinds of robots.") }\end{array}$ & 17 \\
\hline O. Other 4 Categories & 68 \\
\hline
\end{tabular}

Categories A-D were positive opinions of the robots themselves. A corresponds to sentences expressing positive opinions and emotions toward the robots' appearance, interaction, intelligence, and so on. B corresponds to sentences expressing expectations and desires for robots and technology in the future. C corresponds to sentences expressing positive attitudes held by children toward robots, described by the children themselves or their parents. D corresponds to sentences such as "I wanted to interact with the robots more".

Categories E-J were negative opinions of the robots themselves. E corresponds to sentences expressing dissatisfaction with and negative opinions of the robots' functions of utterance, recognition, communication, and so on. F corresponds to sentences expressing negative emotions toward robots, such as anxiety, fear, and so on. G corresponds to sentences stating that children felt fear or anxiety toward the robots, as written by the children themselves or their parents. 
$\mathrm{H}$ corresponds to sentences indicating that children were indifferent to or had no interest in the robots, as written by the children themselves or their parents. I corresponds to sentences expressing other dissatisfaction with the robots. J corresponds to sentences about physical danger in interaction with the robots, such as the fact that a robot's arm struck the visitor's body.

Categories $\mathrm{K}$ and $\mathrm{L}$ were opinions on the exhibition. $\mathrm{K}$ corresponds to a positive evaluation, such as "I would like to visit here again". L corresponds to critical requests about the contents of the exhibition, such as types of robots to be exhibited. The other four categories correspond to sentences on dissatisfaction with external factors not related to the robots or content of the exhibition, such as congestion of the floor and waiting time for demonstrations. Thus, these four categories were reduced in analysis.

Respondents of categories $\mathrm{A}-\mathrm{D}$ and $\mathrm{K}$ were grouped as those having positive opinions, and respondents of categories $\mathrm{E}-\mathrm{J}$ and $\mathrm{L}$ as those having negative opinions. The number of positive opinions and that of negative opinions were $91(31 \%)$ and $134(45.7 \%)$, respectively. The opinions classified into $\mathrm{A}$ and $\mathrm{K}$ dominated more than half of the positive opinions. Moreover, category $\mathrm{E}$ had the largest number of opinions among the negative opinions and acounted for $44 \%$ of the negative opinions.

In order to investigate the relationship between these opinions and the time that the respondents stayed near the robots, a one-way ANOVA with the opinion categories was executed for the behavior index T3. Since the external factor of congestion may influence the analysis, as mentioned in section 3.2 this ANOVA was limited to the respondents on the non-congested days. Figure 5 (b) shows the mean values and standard deviations of T3 on the categories. As a result, there was a statistically significant effect of the categories $(F=2.930, p=0.002)$. A Tukey post-hoc test found that the T3 values of the respondents classified into $\mathrm{C}$ were larger than those in all the other categories except for $\mathrm{H}$.

\subsection{Discussion}

Influence of Age: The results presented in section 3.1 show that many visitors positively evaluated the robots. More specifically, many visitors had interest in and felt friendliness toward the robots. Moreover, many visitors did not feel anxiety about interaction with the robots and their social influence.

On the other hand, there were differences in these opinions between ages. The results show that people in their 20's feel less friendliness toward robots than those in their 30's - 60's, people in their 20's less positively evaluate guidance by the robots than those in their 10's and 60's, people in their 10's more positively evaluate the guidance than those in their 30's and 40's, people in their 10 's feel less anxiety about the social influence of the robots than those in their 20's, 60 's, and 70's, and people in their 40's feel less anxiety about the social influence than those in their 20's and 30's. In other words, younger respondents do not necessarily like the robots more than elderly respondents. The above results imply that the design of robots should be changed according to user age. 
Relationship to Behaviors: The results presented in section 3.2 show that some external factors influence concrete behaviors in real situations, such as museums. However, the results in section 3.3 show that there is no relationship between opinions of the robots and the concrete behavior of staying near the robots. These results imply that environmental factors may more strongly affect behaviors than psychogical factors in real situations such as museums.

Moreover, the results in section 3.3 also show that interest in, friendliness toward, and evaluation of effectiveness of the robots do not necessarily conflict with anxiety toward them. These results imply that robot designs intended to increase effectiveness and friendliness do not necessarily reduce the anxiety felt toward the robot.

Attitudes of Children Toward Robots: The results given in section 3.4 indicate that there are both positive and negative opinions of the robots and the exhibitions on a concrete level. They also show that there exist several areas of dissatisfaction with the functions of the communication robots, and people, in particular children, may have negative emotions toward the robots at their current level. On the other hand, the results also show that there are children who had interest in and friendliness toward the robots and indicate that these children and their parents stay near the robots longer than others.

The above results can be interpreted as follows. In Japan, there are several types of discourses on robots, and their effect naturally differs between age groups. The results in section 3.1 reflect this. Moreover, many children have never seen actual moving robots, although they are affected by several media. This gap may lead to the fear and anxiety toward the robots shown in section 3.4. If this interpretation is valid, we can conclude that the design of robots for children should be adapted for the image of robots presented in the various media.

Gender Difference: The results in section 3.2 reveal a tendency for females to remain near the robots longer than males. However, there is some doubt as to whether there is a gender difference in behavior toward the robots, as shown in section 3.2. at least in the situation presented in this research. In fact, there was no gender difference in opinions shown for the items, nor any correlation between them and the behavior index.

As a reason, it can be surmised that many of the visitors were females in their 30 's and 40's. The period included the summer holidays, and, as a result, many females visited the exhibition with their children. In other words, it can be assumed that their children stayed near the robots longer with them and, as a result, the females appeared to be staying longer. This assumption needs to be investigated through another type of data, such as orbits in which the visitors moved while viewing the exhibits. Such data will be gathered and analyzed in future research.

\section{Summary}

This paper reported the results of questionnaire-based research conducted at an exhibition of interactive humanoid robots that was held at the Osaka Science 
Museum, Japan, with the aim of investigating the use of communication robots connected with a ubiquitous sensor network. More than ninety thousand people visited the exhibition and a questionnaire was given to the visitors to explore their opinions of the robots. Statistical analysis was done for data consisting of 2,301 respondents. It was found that the visitors' opinions of the robots differed according to age, younger people did not necessarily like the robots more than elderly people, positive evaluation of the robots did not necessarily conflict with negative evaluations such as anxiety, there was no gender difference in opinions of the robots, and there was almost no correlation between the opinions and the length of time spent near the robots.

As future research, the relationship between the visitors' opinions of the robots and another behavior index should be explored. Moreover, there was a bias of respondents in assembling samples. Although this bias may be unavoidable in situations such as museums, data from various types of people need to be gathered for analysis.

\section{Acknowledgments}

This research was supported by the Ministry of Internal Affairs and Communications. We wish to thank the staff at the Osaka Science Museum for their highly appreciated cooperation and helpful suggestions: Hideaki Terauchi, Toshihiko Shibata, Koutarou Hayashi, Masaaki Kakio, Taichi Tajika, and Fumitaka Yamaoka.

\section{References}

1. Ng, K.C., Ishiguro, H., Trivedi, M.M., Sogo, T.: An integrates surveillance systemhuman tracking and view synthesis using multiple omni-directional vision sensors. Image and Vision Computing Journal 22 (2004) 551-561

2. Ikeda, T., Ishida, T., Ishiguro, H.: Framework of distributed audition. In: Proc. 13th IEEE Int. Workshop on Robot and Human Interactive Communication (ROMAN). (2004)

3. Murakita, T., Ikeda, T., Ishiguro, H.: Human tracking using floor sensors based on the Markov chain Monte Carlo method. In: Proc. Int. Conf. Pattern Recognition (ICPR). (2004) 917-920

4. Nishimura, T., Itoh, H., Nakamura, Y., Yamamoto, Y., Nakashima, H.: A compact battery-less information terminal for real world interaction. In: PERVASIVE 2004. Number 3001 in LNCS. Springer (2004) 124-139

5. Sumi, Y., Matsuguchi, T., Ito, S., Fels, S., Mase, K.: Collaborative capturing of interactions by multiple sensors. In: Proc. Int. Conf. Ubiquitous Computing (Ubicomp). (2003) 193-194

6. Schulz, D., Fox, D., Hightower, J.: People tracking with anonymous and id-sensors using rao-blackwellised particle filters. In: Proc. Int. Joint Conf. Artificial Intelligence (IJCAI). (2003) 921-926

7. Burgard, W., Cremers, A.B., Fox, D., Hähnel, D., Lakemeyer, G., Schulz, D., Steiner, W., Thrun, S.: The interactive museum tour-guide robot. In: Proc. Nat. Conf. Artificial Intelligence (AAAI). (1998) 
8. Ishiguro, H., Ono, T., Imai, M., Maeda, T., Kanda, T., Nakatsu, R.: Robovie: an interactive humanoid robot. Int. J. Industrial Robot 28 (2001) 498-503

9. Shibata, T., Wada, K., Tanie, K.: Tabulation and analysis of questionnaire results of subjective evaluation of seal robot at Science Museum in London. In: Proc. Int. Workshop on Robot and Human Interactive Communication (RO-MAN). (2002) 23-28

10. Shibata, T., Wada, K., Tanie, K.: Subjective evaluation of a seal robot at the national museum of science and technology in Stockholm. In: Proc. Int. Workshop on Robot and Human Interactive Communication (RO-MAN). (2003) 397-407

11. Shibata, T., Wada, K., Tanie, K.: Subjective evaluation of a seal robot in Burunei. In: Proc. Int. Workshop on Robot and Human Interactive Communication (ROMAN). (2004) 135-140

12. Nomura, T., Kanda, T., Suzuki, T., Kato, K.: Psychology in human-robot communication: An attempt through investigation of negative attitudes and anxiety toward robots. In: Proc. the 13th IEEE International Workshop on Robot and Human Interactive Communication. (2004) 35-40 